\title{
Disturbed Anastomotic Healing after Esophagectomy: A Novel Treatment of a Benign Tracheo-Neo-Esophageal Fistula
}

\author{
C.J. Buskens ${ }^{\mathrm{a}}$ F. van Coevorden ${ }^{\mathrm{b}}$ H. Obertop ${ }^{\mathrm{a}}$ J J.J.B. van Lanschot ${ }^{\mathrm{a}}$ \\ Departments of Surgery, a Academic Medical Center, University of Amsterdam and \\ bThe Netherlands Cancer Institute, Antoni van Leeuwenhoek Hospital Amsterdam, The Netherlands
}

\section{Key Words}

Fistula · Esophagectomy · Trachea

\begin{abstract}
In this report a 65-year-old man is presented who developed a benign tracheo-neo-esophageal fistula 1 month after esophageal resection due to confined leakage of the cervical anastomosis. After unsuccessful conservative treatment, the fistula was partly excised via a cervical approach and a T-drain was inserted in the esophagus, thus creating a new fistula to the skin. Over a period of 8 weeks, the long mediastinal fistula track obliterated, and after removal of the T-drain the (neo-)esophagocutaneous fistula closed rapidly.
\end{abstract}

Copyright @2002 S. Karger AG, Basel

\section{Introduction}

Benign tracheo-neo-esophageal fistulas are a rare but serious complication after esophageal resection. Although the clinical presentation varies, they are frequently lifethreatening [1]. Despite various possible treatment strategies described in the literature (i.e. conservative, endo- scopic, and operation), treatment of such fistulas remains a challenge [2-4].

We present a patient who developed a fistula between the cervical anastomosis and the left main-stem bronchus due to anastomotic leakage after esophagectomy for a carcinoma. Different causes, clinical features and treatment options of this severe complication will be discussed.

\section{Case Report}

A 65-year-old man presented with a 4-month history of dysphagia, odynophagia and considerable weight loss. Endoscopy showed an irregularity in the cardia infiltrating into the distal esophagus, which was suspicious for a malignancy. On biopsies, the pathological diagnosis of a moderately differentiated adenocarcinoma could be established. Endosonography showed invasion of the tumor into the serosa, and multiple enlarged lymph nodes near the celiac trunk (uT3N1Mx). Additional preoperative analysis did not show distant metastases.

After four courses of neoadjuvant chemotherapy (epirubicin, cisplatin and continuous infusion of 5-fluorouracil), the patient underwent transhiatal esophagectomy and gastric tube reconstruction with a right-sided cervical anastomosis. Histological examination of the resection specimen did not show invasive tumor except for some submucosal tumor embolisms. None of the in size decreased lymph nodes were tumor-positive. A radical resection (R0) had been achieved.

\begin{tabular}{ll}
\hline KARGER & ( ) 2002 S. Karger AG, Basel \\
Fax +4161306 12 34 & 0253-4886/02/0192-0088 \$18.50/0 \\
$\begin{array}{l}\text { E-Mail karger@karger.ch } \\
\text { www.karger.com }\end{array}$ & $\begin{array}{l}\text { Accessible online at: } \\
\text { www.karger.com/journals/dsu }\end{array}$
\end{tabular}

C.J. Buskens, MD

Academic Medical Center, University of Amsterdam

Suite G4-130, Meibergdreef 9

NL-1105 AZ Amsterdam (The Netherlands)

Tel. +31 20 5669111, Fax +3120 6914858, E-Mail C.J.Buskens@amc.uva.nl 
Fig. 1. Oral contrast study showing an irregular mediastinal cavity ending into a fistula to the left main-stem bronchus.

Fig. 2. Gastroscopic view showing a vital gastric tube with incomplete healing of the cervical anastomosis at the lateral side. * Opening of fistula.
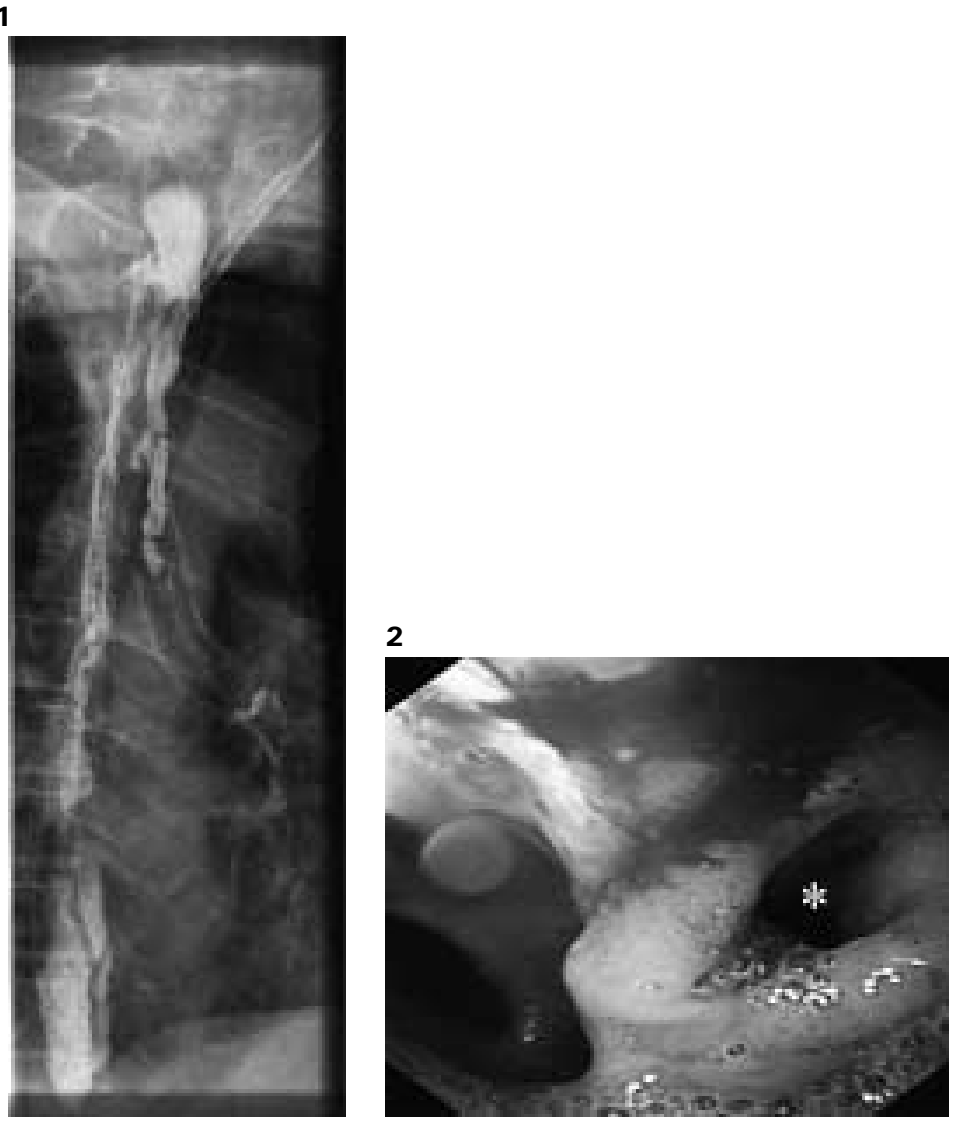

After 1 week a routine oral contrast study did not show leakage of the cervical anastomosis and oral feeding was resumed. However, 10 days later the patient developed fever and difficulties with swallowing. Anastomotic leakage to the mediastinum was now seen on a repeated oral contrast study. With conservative treatment (i.e. nil per mouth, jejunostomy feeding and antibiotics) the leakage closed, but another 3 weeks later a long fistula between the cervical anastomosis and the left main-stem bronchus had developed (fig. 1). At bronchoscopy a 2- to 3-mm defect on the dorsal side of the left main-stem bronchus was seen, just below the tracheal bifurcation. Gastroscopy showed a vital gastric tube with incomplete healing of the cervical anastomosis at the left lateral side (fig. 2).

With conservative treatment the patient deteriorated and another 10 days later, it was decided that surgical intervention was inevitable. The patient was transferred to the Academic Medical Center. Via a left-sided cervical incision, the anastomotic defect was identified. The fistula was partly excised and a T-drain was inserted into the esophagus, in order to create a new fistula to the skin, leaving the long, mediastinal track to the left bronchus to obliterate (fig. 3a, b). A second silastic drain was placed in the mediastinal cavity to guarantee sufficient drainage. Step by step this last drain was withdrawn from the cavity. After 2 months the fistula to the left main bronchus and the mediastinal cavity had closed completely. After removal of the T-drain, the (neo-)esophago-cutaneous fistula closed rapidly.

Four months later, the patient is now capable of sufficient oral intake without swallowing difficulties.

\section{Discussion}

A patient is presented with a benign tracheo-neoesophageal fistula 1 month after esophageal resection, which is a rare complication of this operation. In a consecutive series of 383 patients who underwent subtotal esophagectomy with a cervical anastomosis, only 1 patient $(0.3 \%)$ developed such a fistula in our institution [4].

In the presented patient a fistula developed due to leakage of the cervical anastomosis with mediastinal abscess formation and secondary fistulization to the trachea, which is probably the most important cause of this complication [5]. Although the development of this complication might be related to preoperative chemotherapy, numbers are still too small to confirm a supposed correlation $[4,5]$.

Other common causes of fistulization are devascularization of the major airways after a transthoracic resection with extensive dissection in the upper mediastinum $[5,6]$, or a direct traumatic injury to the trachea, especially after blunt transhiatal resection [7]. 
Fig. 3. a Radiological contrast study via inserted T-drain showing an irregular mediastinal cavity with the cervical T-drain into the gastric tube in situ. b Schematic diagram of the cervical T-drain in the gastric tube inducing re-routing of the fistula from the major airways to the skin, and the second drain in the mediastinal cavity for the purpose of flushing. $1=$ Cervical esophagus; $2=$ cervical anastomosis with incomplete healing; 3 = gastric tube; $4=$ trachea; $5=$ left main bronchus; $6=$ mediastinal cavity $7=$ fistula track to left bronchus; $8=$ T-tube into (neo-)esophagus; 9 = drain into mediastinal abscess.
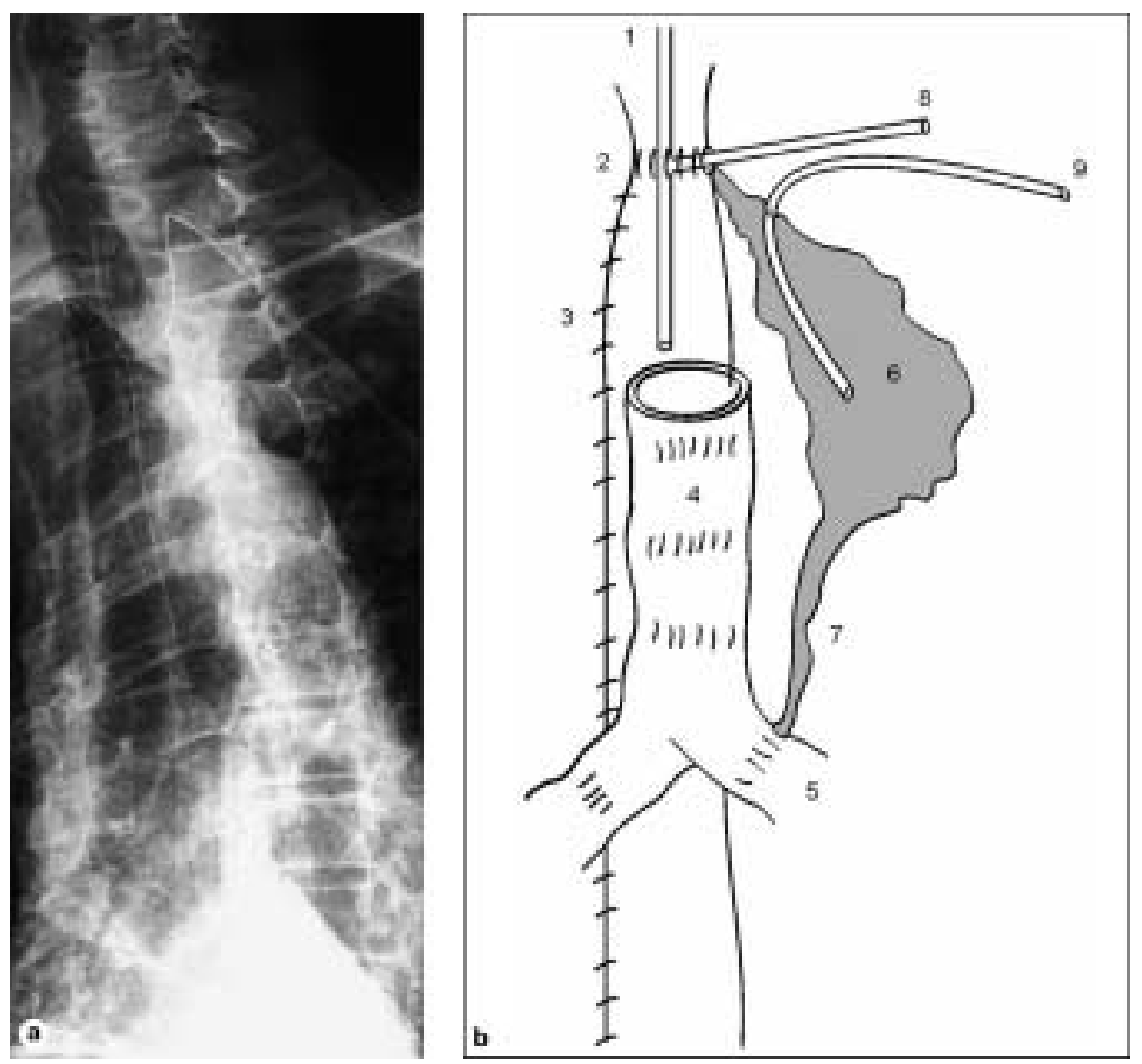

The nature of the signs and symptoms varies and is predominantly determined by the site and size of these fistulas. The most common symptoms are mild cough associated with oral intake and weight loss, but patients can also present with more severe recurrent bronchopneumonia and even a life-threatening mediastinitis $[1,8]$.

Early diagnosis is important and often not too difficult. Radiological examination with oral contrast can usually confirm the diagnosis when a fistula is suspected, although often a false positive bronchogram is seen after aspiration of contrast during the investigation (e.g. due to recurrent nerve paresis). Additionally, this may be combined with upper gastrointestinal endoscopy and bronchoscopy to characterize the fistula more precisely.

There is still considerable diversity of opinion regarding the optimal treatment of this lesion [2-4], which is at least partly due to the fact that it is a rare event. In the literature, an operative approach is mostly advocated, but in patients with only mild symptoms, a conservative treatment regimen (i.e. nil per mouth, artificial enteral feeding, with or without antimicrobial agents) may be considered [2].
Endoscopic obliteration is another option, especially in patients with mild symptoms and a long fistula track. With fibrin glue or hemoclips, permanent closure of the fistula can be achieved, but this technique may require several attempts and is predominantly described in children with a tracheo-esophageal fistula after surgery for esophageal atresia [9].

When conservative treatment fails or symptoms are more severe, a surgical intervention is necessary. A direct approach with closure of the tracheal and esophageal defects is preferred. Additionally, vital tissue (e.g. a muscle flap) can be applied to the defect to fill the dead space and prevent recurrent fistulization [10]. However, this technique is often complicated in patients who underwent recent esophagectomy and puts the neo-esophagus at risk of increased damage.

We describe a novel technique of a local cervical approach, which is specifically suitable for patients with a long fistula track. It prevents a (re-)thoracotomy and saves the neo-esophagus. Partial excision of the fistula track and adequate external drainage of a cervico-mediastinal cavity are essential. A cervical T-tube into the (neo-)esopha- 
gus induces re-routing of the fistula from the major airways to the skin. The created cutaneous salivary fistula will rapidly close after removal of the T-drain.

However, if the gastric tube cannot be preserved (e.g. because of partial necrosis), continuity of the gastrointestinal tract can be restored during the same operation with a colonic interposition $[11,12]$. Under these circumstances, the colon segment is preferably placed in a prevertebral position to reinforce the posterior wall of the trachea, but if a distal remnant of the gastric tube is left in situ, a combination of a distal retrosternal and proximal prevertebral colonic interposition can be created [4].

Especially in patients with severe mediastinitis, it can be necessary to create a temporary esophagostomy with reconstruction of the gastrointestinal tract at a later stage. When the septic focus has been effectively eliminated, the fistula has closed, and the patient has recovered, an extra-anatomic (i.e. retrosternal or subcutaneous) colonic interposition can be created thus avoiding a redo operation in the previously inflamed prevertebral compartment [4].

A benign tracheo-neo-esophageal fistula following esophagectomy is a rare but serious and challenging complication. It is predominantly the site, size and underlying cause in combination with the severity of symptoms which should determine the treatment strategy. Conservative treatment can be justified, but in patients with a persisting fistula track or in the presence of severe symptoms, surgical intervention, with a tailored approach, is inevitable. With an individualized treatment, this potentially life-threatening condition can be managed successfully.

\section{References}

1 Gudovsky LM, Koroleva NS, Biryukov YB, Chernousov AF, Perelman MI: Tracheoesophageal fistulas. Ann Thorac Surg 1993;55:868875.

2 Semlacher RA, Bharadwaj BB, Nixon JA: Management of post-traumatic tracheo-esophageal fistula following failed primary repair. J Thorac Cardiovasc Surg 1994;35:83-86.

3 Baisi A, Bonavina L, Narne S, Peracchia A: Benign tracheoesophageal fistula: Results of surgical therapy. Dis Esophagus 1999;12:209_ 211.

4 Buskens CJ, Hulscher JBF, Fockens P, Obertop $\mathrm{H}$, Van Lanschot JJB: Benign tracheo-neoesophageal fistulas after subtotal esophagectomy. Ann Thorac Surg 2001;72:221-224.
5 Bartels HE, Stein HJ, Siewert JR: Tracheobronchial lesions following oesophagectomy: Prevalence, predisposing factors and outcome. Br J Surg 1998;85:403-406.

6 Fujita H, Kawahara H, Hidaka M, Nagano T, Yoshimatsu H: An experimental study on viability of the devascularized trachea. Jpn J Surg 1988; 18:77-83.

7 Hulscher JBF, Ter Hofstede E, Kloek J, Obertop H, De Haan P, Van Lanschot JJB: Injury to the major airways during subtotal esophagectomy: Incidence, management, and sequelae. $\mathrm{J}$ Thorac Cardiovasc Surg 2000;120:1093-1096.

8 Wychulis AR, Ellis FHJ, Andersen HA: Acquired nonmalignant esophagotracheobronchial fistula. Report of 36 cases. JAMA 1966; 196:117-122.
9 Ng WT, Luk HT, Lau CW: Endoscopic treatment of recurrent tracheo-oesophageal fistulae: The optimal technique. Pediatr Surg Int 1999; 15:449-450.

10 Hayashi K, Ando N, Ozawa S, Tsujizuka K, Kitajima M, Kaneko T: Gastric tube-to-tracheal fistula closed with a latissimus dorsi myocutaneous flap. Ann Thorac Surg 1999;68:561562.

11 DeMeester TR, Johansson KE, Franze I, et al: Indications, surgical technique and long-term functional results of colon interposition or bypass. Ann Surg 1988;208:460-474.

12 Furst H, Hartl WH, Lohe F, Schildberg FW: Colon interposition for esophageal replacement: An alternative technique based on the use of the right colon. Ann Surg 2000;231:173178. 